\title{
Fortschritte im Controlling durch Business Forecasting
}

Oscar A. G. Treyer

\section{Einleitung}

Das strategische Management erfährt momentan aufgrund zunehmender Globalisierung, verbunden mit intensiverem Wettbewerb und dem steigenden Kampf um die Investorengunst eine neue Dimension. Durch den Sarbanes-Oxley Act (vgl. SEC 2003) erhält die Verantwortlichkeit für den Chief Financial Officer (CFO) und den Chief Executive Officer (CEO) einen neuen Stellenwert. Obwohl sich diese Verantwortlichkeit heute mehrheitlich in „formalen“ Richtlinien widerspiegelt, muss die Verbesserung der Entscheidungsqualität weiterhin das Hauptanliegen sein.

Der Beitrag geht der Frage nach, welche Möglichkeiten ein systematisches Business Forecasting zur „inhaltlichen" Verbesserung der Entscheidungsqualität des Unternehmens liefern kann.

Unter Business Forecasting versteht man traditionell (vgl. Hanke/Wichern 2005, S. 1) die Verbesserung von Unternehmensentscheidungen durch Eingrenzung der Prognose-Unsicherheiten anhand methodisch stringenter Vorgehensweise, meist aufgrund von Erkenntnissen aus Daten-Mustern der Vergangenheit.

\section{Problemstellung}

Üblicherweise beurteilt der Investor (Shareholder) die Performance des Unternehmens mittels externer Finanzberichterstattung. Der Benchmark der Perfor-
mance-Messung lautet meist gegenüber "Vorjahr“ oder gegenüber der „durchschnittlichen Erwartung der Finanzanalysten".

Der Benchmark „Vorjahr" entspricht der sogenannten „naiven “ Prognose. Obwohl diese methodisch einfach ist, enthält sie de facto keinen Erkenntniswert. Sie wird aber selten in Frage gestellt, da sie eine Kompromisslösung bzw. „neutrale Zone" zwischen höher und niedriger darstellt: Kein Unternehmen wird üblicherweise einen Benchmark unter dem Vorjahreswert anstreben, da dies als nicht kompetent eingestuft wird; kein Unternehmen wird einen Benchmark (weit) über dem Vorjahreswert festsetzen, da es sich damit - im heutigen Wirtschaftsumfeld - nur einem unnötigen Risiko der Ziel-Nicht-Erreichung aussetzt.

Der Benchmark „durchschnittliche Erwartung der Finanzanalysten " ist eine andere Form der Kompromisslösung, die aber in Anbetracht der Affären um WorldCom und Enron zunehmend unter Beschuss gekommen ist. Zudem wird von einem Benchmark unter anderem verlangt (vgl. Reilly/Brown 2003, S. 1140), dass er nicht mehrdeutig (unambiguous) sein dürfe. Die Nachvollziehbarkeit ist aber bei der durchschnittlichen Erwartung der Finanzanalysten nicht gegeben.

Bei den beiden oben aufgeführten Benchmarks stellt sich für den Investor das Problem, sich zwischen Bedeutsam-

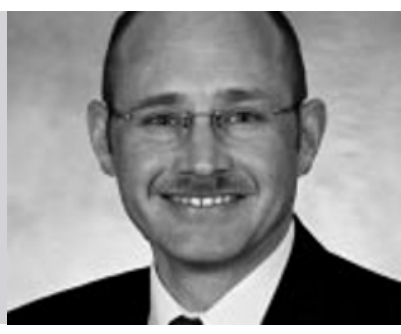

Dr. oec. publ. Oscar A. G. Treyer Universität St. Gallen, Ständiger Dozent, Institut für Accounting, Controlling und Auditing Rosenbergstrasse 52, CH -9000 St. Gallen oscar.treyer@unisg.ch
- Die Verantwortlichkeit des Managements bekommt durch den Sarbanes-Oxley Act (SOX) einen neuen Stellenwert. - Die Verbesserung der "inhaltlichen" Entscheidungsqualität bleibt weiterhin das Hauptanliegen des Controllings. - Der Beitrag zeigt Möglichkeiten auf, wie anhand eines systematischen Business Forecasting eine Verbesserung der "inhaltlichen" Entscheidungsqualität mit mehr Bedeutsamkeit (relevance), ohne Abstriche an der Zuverlässigkeit (reliability), erreicht werden kann. - Dazu bietet sich zweckmäßigerweise die Budgetierung als Benchmark der PerformanceMessung an.

- Die dabei verfolgten zwei Ebenen betreffen "Better Budgeting" sowie "Beyond Budgeting".

keit (relevance) und Verlässlichkeit (reliability) zu entscheiden: Dem Benchmark „Vorjahr" fehlt durch den nicht vorhandenen Erkenntniswert die entsprechende Bedeutsamkeit, dem Benchmark „durchschnittliche Erwartung der Finanzanalysten “ durch seine Mehrdeutigkeit die notwendige Verlässlichkeit.

In den folgenden Ausführungen wird die enge Perspektive des Investors verlassen und auf die Unternehmenssicht fokussiert. Dabei wird untersucht, wie das Unternehmen und dessen Management mehr Bedeutsamkeit, ohne Abstriche an der Verlässlichkeit, durch Implementierung eines systematisches Business Forecasting erreichen kann. Dazu bietet sich zweckmäßigerweise die Budgetierung als Benchmark der Performance-Messung an. 


\section{Implikationen für die strate- gische und operative Führung}

Obwohl in letzter Zeit die Budgetierung nicht mehr als ein wichtiger Koordinationsprozess betrachtet wird, sondern eher als ein dysfunktionales Disziplinierungs-Instrument, sollte nicht vergessen werden, dass eines der Grundgesetze menschlichen Handelns wie folgt zusammengefasst werden kann: „What gets measured gets done“. Viele der meist berechtigten, negativen Auswirkungen der heutigen Budgetierungspraxis, bzw. der Budgetierung als „fixierter Leistungsvertrag" (vgl. Hope/Fraser 2003, S. 10 ff.), können durch den Einsatz von Techniken des Business Forecasting „neutralisiert" werden. Das Business Forecasting kann diesbezüglich auf zwei Stufen einen Beitrag leisten, nämlich auf der Ebene „Better Budgeting" sowie auf der Ebene „Beyond Budgeting“.

\section{Ebene „Better Budgeting”}

Einer der Hauptkritikpunkte der Budgetierung als „fixierter Leistungsvertrag“ besteht darin, dass die Budgetvorgaben einen geringen Realitätsbezug aufweisen. Dadurch werden weder das Verhalten (vgl. Fickert 2004, S. 712 f.), noch die Motivationswirkung (vgl. Ewert/Wagenhofer 2005, S. 417 ff.) berücksichtigt.

(Forecast-) Vorgaben haben aber nur dann einen positiven Einfluss auf das Verhalten bzw. die Motivation, wenn sie als wirklichkeitsnah eingestuft werden. Es ist deshalb überaus wichtig, ungesehen davon wie hoch oder ambitiös die Ziele festgelegt werden, dass die „Saisonalität" des Unternehmensumfeldes bei Budgetvorgaben berücksichtigt wird.

Um dies zu erreichen, bietet die Statistik üblicherweise die Technik der sogenannten „klassischen" Zeitreibenzerlegung an (vgl. Makridakis/Wheelwright/ Hyndman 1998, S. 106 ff. und Siegel 2003, S. 626 ff.). Dabei werden entsprechend der Saisonalität (z. B. Monat) die durchschnittlichen Abweichungen gegenüber einer gewählten „Basis“ (z. B. gleitender Durchschnitt) berechnet. Anhand dieser „Saisonkomponenten“ ist es mög-

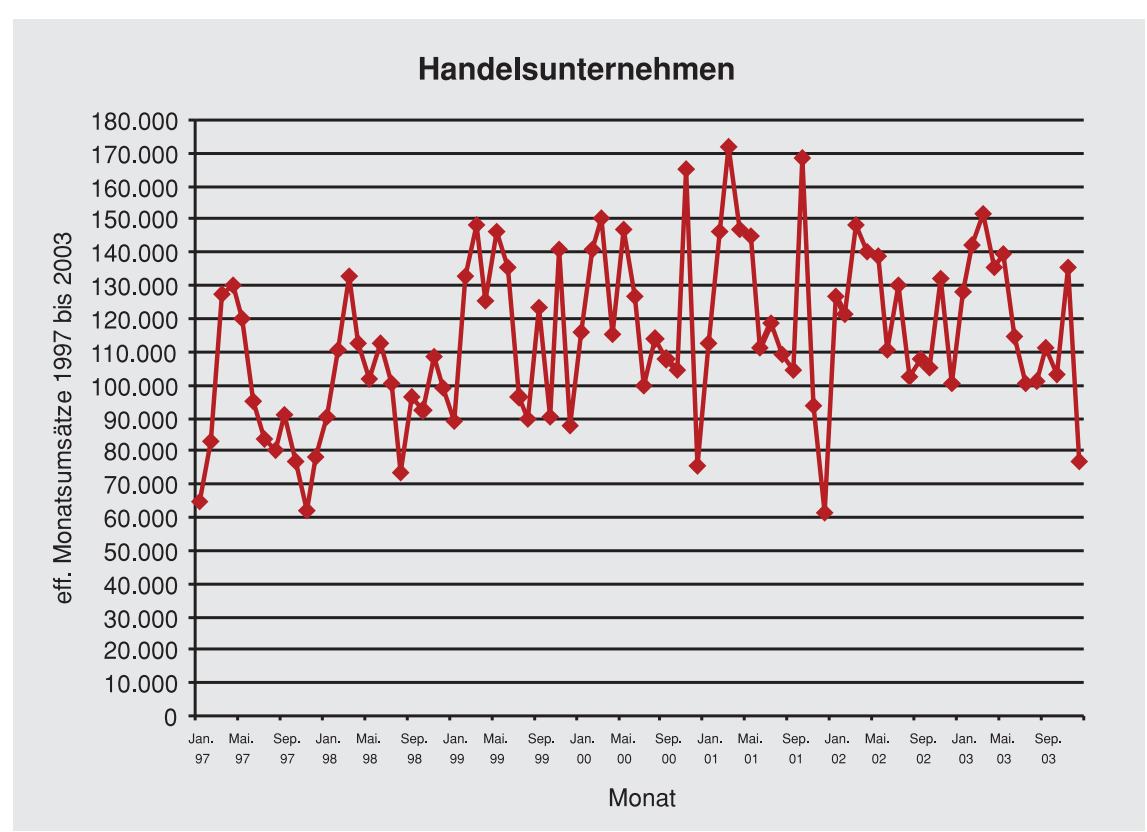

Abbildung 1: Effektive Monatsumsätze 1997 bis 2003

lich, die Budgetvorgaben zu saisonalisieren.

Zu Illustrationszwecken werden in der Folge die effektiven Monatsumsätze eines mittelständischen Han-

\begin{tabular}{l|c|l|r}
\hline Januar & $-5.321,20$ & Juli & $-8.717,85$ \\
\hline Februar & $16.297,41$ & August & $-19.480,77$ \\
\hline März & $34.126,36$ & September & $-9.879,13$ \\
\hline April & $12.613,00$ & Oktober & $-8.892,59$ \\
\hline Mai & $18.901,32$ & November & $1.732,69$ \\
\hline Juni & 591,53 & Dezember & $-31.970,77$ \\
\hline
\end{tabular}
delsunternehmens

Abbildung 2: Saisonkomponenten anhand „klassischer" Zeitreihenzerlegung für die Jahre 1997 bis 2003 herangezogen (vgl. Abb. 1).

Anhand der „klassischen“ Zeitreihenzerlegung (additives Modell zu gleitendem Durchschnitt) können die (absoluten) Saisonkomponenten für das Handelsunternehmen ermittelt werden (vgl. Abb. 2):

Für das Controlling stellen sich hier zwei Fragen, nämlich welche Signifikanz bzw. „Güte“ weisen diese Saisonkomponenten auf und welche „Basis“ ist zu wählen, damit eine konsistente Umsetzung möglich wird.

\section{Frage der Signifikanz bzw. „Güte"}

Die anhand der „klassischen “ Zeitreibenzerlegung berechneten Saisonkomponenten (vgl. Abb. 2) sind „einfache“, durchschnittliche Abweichungen. Über die
„Treffsicherheit" dieser Angaben kann aber keine Aussage gemacht werden.

Das Business Forecasting bietet hierfür eine elegante Lösung an, nämlich die multiple Regression mit Dummy-Variablen für Saisonkomponenten (vgl. Hankel Wichern 2005, S. $349 \mathrm{ff}$. und Wilson/ Keating/Galt 2002, S. 213 ff.).

Für das Handelsunternehmen ergibt dies die in Abbildung 3 aufgeführte "Gleichung“.

Das Spezielle an dieser Lösung (vgl. Abb. 3) besteht darin, dass der Achsenabschnitt C(1) die (absolute) Saisonkomponente des Monats Januar, der Regressionskoeffizient $\mathrm{C}(2)$ die Steigung des Trends " $\mathrm{T}$ “ und alle folgenden Regressionskoeffizienten C(3) bis C(13) die (absoluten) Abweichungen der Saisonkomponenten Februar bis Dezember zur Sai- 
effektive Monatsumsätze $=$

$C(1)+C(2) \cdot T+C(3) \cdot M 2+C(4) \cdot M 3+C(5) \cdot M 4+C(6) \cdot M 5+C(7) \cdot M 6+C(8) \cdot$ $M 7+C(9) \cdot M 8+C(10) \cdot M 9+C(11) \cdot M 10+C(12) \cdot M 11+C(13) \cdot M 12$

Abbildung 3: "Gleichung" multiple Regression mit Dummy-Variablen verbessern, nicht aber eine $100 \%$ ige „Erklärung“ abgegeben werden kann. Weiter kann ausgesagt werden, dass die Abweichungen „effektive Werte mi-

sonkomponente des Monats Januar darstellen.

Die „statistische“ Auswertung für das Handelsunternehmen gemäß obiger "Gleichung“ ist in Abbildung 4 aufgeführt.

Mit dem adjustierten Bestimmtheitsmaß ist bereits eine Grobeinschätzung der „Güte“ der multiplen Regression (vgl. Abb. 3) möglich: Im vorliegenden Fall bedeutet dies, dass rund $55 \%$ der „Abweichungen“ durch das Modell „erklärt“, bzw. rund $45 \%$ nicht durch die "Gleichung " erfasst werden. In einem weiteren Schritt können anhand der t-Statistiken bzw. p-Werte gemäss der üblichen Regressionsanalyse klare Signifikanz-Aussagen gemacht werden: Die Saisonkomponenten für Januar [C] bis Mai [M5] sowie Dezember [M12] sind statistisch signifikant ( $\mathrm{t}$-Statistiken der Regressionskoeffizienten klar über 2.0, p-Wert klar unter 0.05 bzw. $5 \%$ ), diejenigen von Juni [M6] bis November [M11] nicht.

Für das Controlling bedeutet dies im vorliegenden Fall, dass die saisonalisierten Budgetvorgaben den Realitätsbezug nus saisonalisierte Budgetvorgaben " für die Monate mit statistisch signifikanten Saisonkomponenten sehr ernst zu nehmen sind und fundiert analysiert werden sollten. Im Gegensatz dazu besteht aber bei den Monaten mit statistisch nicht signifikanten Saisonkomponenten eine entsprechend hohe Wahrscheinlichkeit, dass die berechnete Saisonalisierung lediglich „zufällig“ entstanden ist. Eine diesbezügliche Abweichung ist somit eindeutig weniger fundiert.

Durch dieses Wissen, welches im Unternehmen kommuniziert werden sollte, ist es dem Controlling möglich, fundierter und prioritätsbezogener seine Funktion wahrzunehmen sowie den Realitätsbezug und die Motivation der Linienverantwortlichen zu steigern.

\section{Frage der umsetzungs- konsistenten "Basis"}

Die „klassische“ Zeitreihenzerlegung geht üblicherweise davon aus, dass die Saisonkomponenten anhand der durchschnittlichen Abweichungen gegenüber dem zentrierten, gleitenden Durchschnitt berechnet werden. Erst auf der Basis der anschließend ermittelten „saisonbereinigten "Werte wird anhand einer einfachen Regression der Trend berechnet.

Die Ermittlung der Saisonkomponenten anhand der multiplen Regression mit Dummy-Variablen geht grundsätzlich von Saison-Abweichun- gen (nach der Methode der kleinsten Quadrate) gegenüber dem im Modell „integrierten“ Trend aus.

Bei beiden Berechnungsmodellen ist aber der „rechnerische“ Trend kaum mit den Budgetvorgaben identisch, da diese das Resultat einer Vielzahl von Input-Faktoren widerspiegeln. Damit das Controlling die Saisonalität auch bei „losgelösten“ Budgetvorgaben konsistent umsetzen kann, muss ein rechnerischer „Kunstgriff“ angewendet werden, der keine wesentlichen Abstriche an der Verlässlichkeit auslöst.

Bei der „klassischen“ Zeitreihenzerlegung besteht eine mögliche praktikable Umsetzungsvariante darin, dass vorerst die Differenz zwischen dem effektiven und budgetierten Jahresumsatz als „Trend“ deklariert und - bei Monats-Saisonalität auf die einzelnen Monate herunter gebrochen wird. Damit die saisonalisierten Monats-Budgetvorgaben ermittelt werden können, wird die Summe aus dem effektiven durchschnittlichen Monatsumsatz zuzüglich dem vorstehend berechneten monatlichen Trend um die jeweilige Saisonkomponente „korrigiert“.

Bei Verwendung der multiplen Regression mit Dummy-Variablen geht man grundsätzlich gleich vor. Man muss aber vorbereitend die Abweichungen der Saisonkomponenten Februar bis Dezember zur Saisonkomponente des Monats Januar in „absolute“ Saisonkomponenten Januar bis Dezember umwandeln.

Das Handelsunternehmen erwirtschaftete im Jahre 2003 einen effektiven Jahresumsatz von 1.440.234. Für das Jahr 2004 setzte das Management einen budgetierten Jahresumsatz von 1.563.800 fest. Basierend auf diesen Ausgangsdaten können die in Abbildung 5 dargestellten saisonalisierten, budgetierten Monatsumsätze 2004 ermittelt werden. Unter Verwendung der oben aufgeführten praktikablen Umsetzungsvariante wurde die Saisonalität konsistent zu den Budgetvorgaben implementiert.

Die Abbildung 5 zeigt klar auf, dass die Saisonalisierung gemäss „klassischer" Zeitreihenzerlegung und gemäss multipler Regression sehr ähnlich verläuft. Wobei - wie bereits vorstehend erwähnt - lediglich bei der Anwendung 
der multiplen Regression eine Aussage bezüglich Signifikanz bzw. „Güte“ gemacht werden kann.

Anhand dieser konsistenten, saisonalisierten Monats-Budgetvorgaben kann das Controlling die Akzeptanz durch die Linienverantwortlichen steigern.

\section{Ebene "Beyond Budgeting"}

Unabhängig, ob die „klassische“ Budgetierung in Frage gestellt wird oder nicht, benötigt jedes Führungsinstrument Benchmarks (z. B. in Form von Key Performance Indicators (KPI's)), die nachvollziehbar sind. Dies kann beispielsweise durch ein sogenanntes „Finanzcockpit“ geschehen (vgl. Fickert 2004, S. 710 ff.). Auch wenn die Nachvollziehbarkeit grundsätzlich gewährleistet ist, gerät man bei der Festlegung der Höhe des Benchmarks sehr schnell in einen Erklärungsnotstand. Dieser kann umgangen werden, indem das „richtige“ Benchmark-Niveau gar nicht zur Diskussion gestellt, sondern das sich in der Vergangenheit reflektierende Zeitreihenmuster als die zu übertreffende Vorgabe herangezogen wird. Sowohl eine positive als auch negative Abweichung von diesem „Vergangenheitsmuster" wird als außerhalb der „normalen“ Entwicklung angesehen. Mit diesem „realistischen“ Frühwarnsystem erhält das Management genau die Führungs-Fokussierung, welche heute verlangt wird.

Das Business Forecasting bietet zur Festlegung dieses Benchmark die Technik des exponentiellen Glättens und die ARIMA-Box-Jenkins-Methode an (vgl. Hanke/Wichern 2005, S. 114 ff. \& $381 \mathrm{ff}$. sowie Wilson/Keating/Galt 2002, S. 104 ff. \& 287 ff. und Makridakis/Wheelwright/ Hyndman 1998, S. $147 \mathrm{ff} . \& 311 \mathrm{ff}$.). Beide Methoden gehen grundsätzlich davon aus, dass die Zeitreihenwerte autokorrelieren, d.h. dass die Werte von heute in einem $\mathrm{Zu}$ sammenhang zu den Werten von gestern stehen. Dabei berücksichtigen sie sowohl die Saisonalität als auch den Trend. Zudem zeigen beide Methoden nicht unmittelbar sogenannte „Strukturbrüche“ in den Zeitreihenwerten auf. Genau dies wird nun genutzt, um Ab-

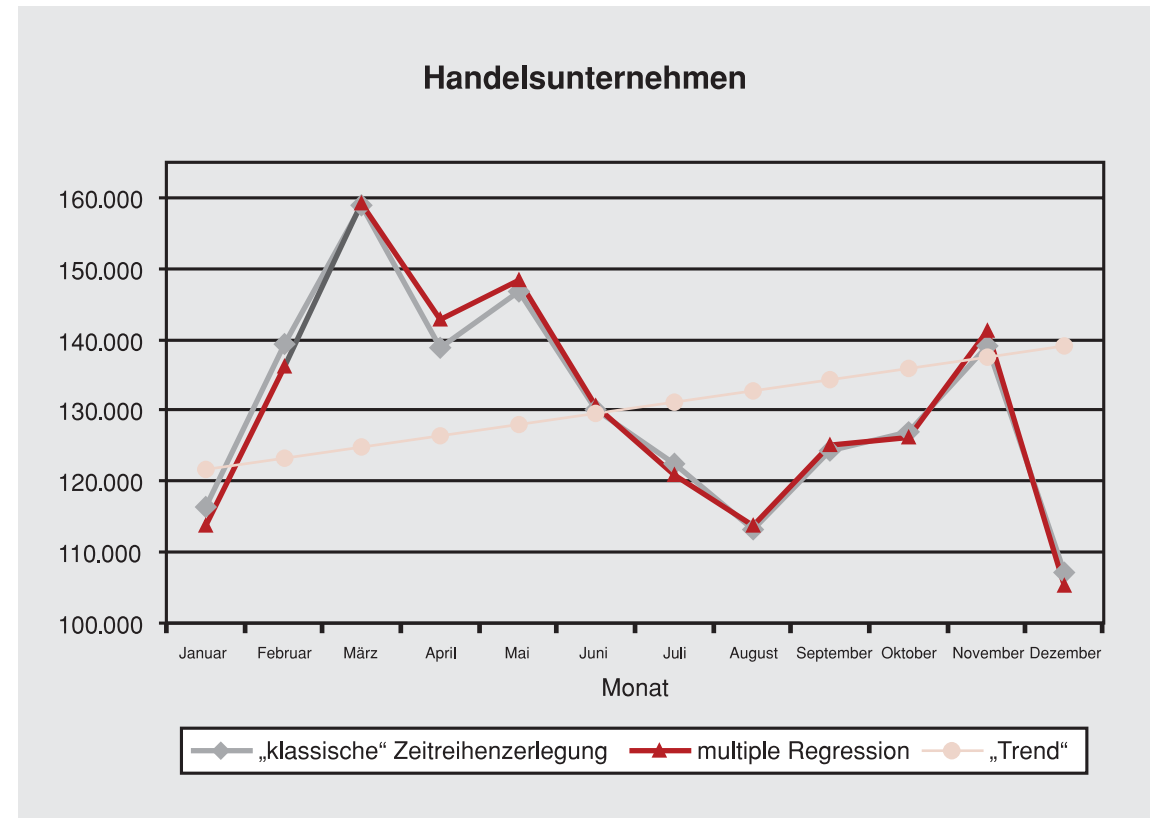

Abbildung 5: Saisonalisierte, budgetierte Monatsumsätze 2004

weichungen der „normalen“ Entwicklung festzustellen.

Die Technik des exponentiellen Glättens geht davon aus, dass der Einfluss der vergangenen Zeitreihenwerte mit zunehmendem „Alter“ exponentiell abnimmt. Da dadurch der letzte, effektive Zeitreihenwert den größten Einfluss auf die Prognose hat, eignet sich diese Technik vor allem für kurzfristige Forecasts.

Die ARIMA-Box-Jenkins-Methode versucht anhand der vergangenen Zeitreihenwerte in einer iterativen Annäherung ein mögliches (Verhaltens-)Modell aus einer Vielzahl von Modellen zu identifizieren. Das gewählte Modell wird dann als geeignet bezeichnet, wenn die Summe der Prognosefehler möglichst klein ist und die einzelnen Residuenabweichungen ein sogenanntes „weißes Rauschen“ (d. h. keine ,sichtbare“ Information) aufweisen. Da das gewählte Modell nicht direkt von den letzten effektiven Werten beeinflusst wird, eignet sich diese Technik auch gut für längerfristige Forecasts.

Anhand der effektiven Monatsumsätze 1997 bis 2003 ist es für das Handelsunternehmen möglich, mit der Technik des exponentiellen Glättens und unter Anwendung der ARIMA-Box-Jenkins-
Methode, die Forecasts für die Monate Januar bis Dezember des Jahres 2004 zu erstellen (vgl. Abb. 6). Die meisten Software-Pakete lassen auch eine Berechnung der oberen und unteren Vertrauensintervall-Grenze zu (hier im 20\% - $80 \%$ Bereich).

Wie die Abbildung 6 zeigt, sind im Falle des Handelsunternehmens die ermittelten Monatsumsatz-Forecasts bei beiden Methoden sehr ähnlich.

Für das Controlling bedeutet dies nun, dass anhand der ermittelten Forecasts „neutrale“ Benchmarks festgelegt wurden. Unabhängig von den Budgetvorgaben kann dadurch eine Aussage gemacht werden, ob das "Vergangenheitsmuster" weiterhin gilt, bzw. ob es über- oder unterschritten wird.

Im vorliegenden Fall des Handelsunternehmens war das Management in den ersten Monaten des Jahres 2004 eher optimistisch, bzw. war überzeugt, dass die im Jahre 2003 lancierten ProduktInnovationen die „auslaufenden“ Produktlinien mehr als kompensierten. Erst im Monat Mai kam Unruhe in der Führungsetage auf: Man unterschritt zum ersten Mal deutlich den MonatsForecast. Entsprechend wurden SparSzenarien aufgestellt, die - bei allfällig 


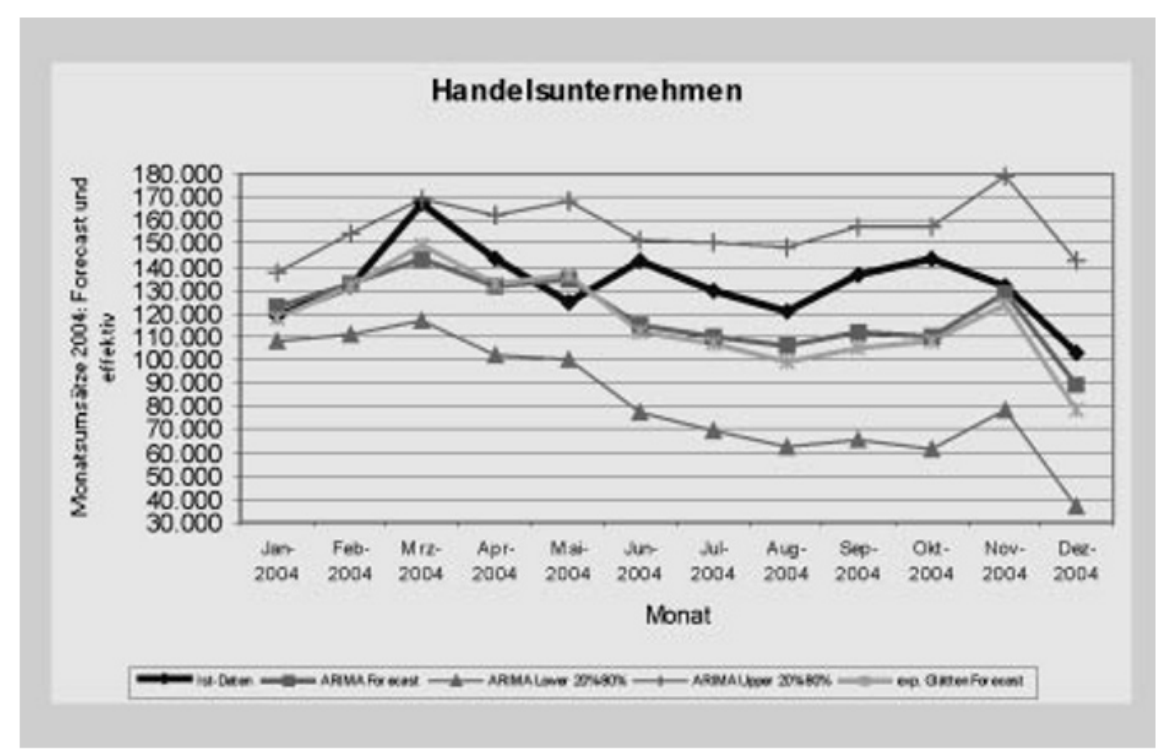

Abbildung 6: Monatsumsätze 2004: Forecast und effektiy

weiter "negativen " Abweichungen - hätten implementiert werden können. Wie die Entwicklung der effektiven Monatsumsätze Juni bis Dezem ber 2004 zeigen, war dies für das Handelsunternehmen glücklicherweise nicht notwendig.

Durch den Einsatz von Tecbniken des Business Forecasting ist es somit möglich, ein Frühwarnsystem ${ }^{\alpha}$ zu implementieren, welches das Management in die Lage versetzt, rechtzeitig und situationsgerecht zu handeln.

\section{Schlussfolgerungen}

Durch die Implementierung eines systematischen Business Forecasting ist es möglich, die Erkenntnisse aus den DatenMustern der Vergangenheit zur Verbesserung der "inhaltlichen" Entscheidungsqualität zu nutzen. Die Bedeutsamkeit (relevance) der Vergangenheit besteht darin, dass jedes Unternehmen - und sei dies lediglich aufgrund ihrer Unternehmenskultur - eine gewisse Remanenz aufweist. Letztere wird nun dazu verwendet, „neutrale " Benchmarks zu ermitteln, wodurch keine Abstriche an der Zuverlässigkeit (reliability) hervorgerufen werden. Das Controlling erhält damit ein wertvolles Instrument, um seiner überparteilichen Lotsenfunktion vermehrt gerecht $z u$ werden.

\section{Literatur}

EWERT, R./WAGENHOFER, A.: Interne Unternehmensrechnung, 6. Aufl., Berlin 2005.

FICKERT, R.: Business Performance und Finanzcontrolling, in: Der Schweizer Treuhänder, 9/04, 2004 , S. $707-714$.

HANKE, J./WICHERN, D.: Business Forecasting, 8. Aufl., Upper Saddle River (NJ) 2005.

HOPE, J.FraSER, R.: Beyond Budgeting, Bost on 2003.

MAKRIDAIIS, S./WHEELWRIGHT, S./HYNDMAN, R.: Forecasting - Methods and Applications, 3. Aufl., New York 1998.

REILLY, F. / BROWN, K.: Investment Analysis and Portfolio Management, 7. Aufl., Mason (Ohio) 2003.

SEC -U.S. Securities and Exchange Commission Final Rule: Management's Reports on Internal Control Over Financial Reporting and Certification of Disclosure in Exchange Act Periodic Reports, Release Nos. 33-8238, 34-47986, IC-26068, File Nos. S7-40-02, S7-06-03, Washington 2003, URL http://www.sec.gov/rules/ final/33-8238.htm.

Siegel, A.: Practical Business Statistics, 5. Aufl., Boston 2003.

WILSON, J./KEATING, B./GaLT, J.: Business Forecasting with Acompanying Excel-Based Forecast $\mathrm{X}^{\mathrm{T} M}$ Software, 4. Aufl., Boston 2002.
State-of-the-Art der Unternehmensbewertung

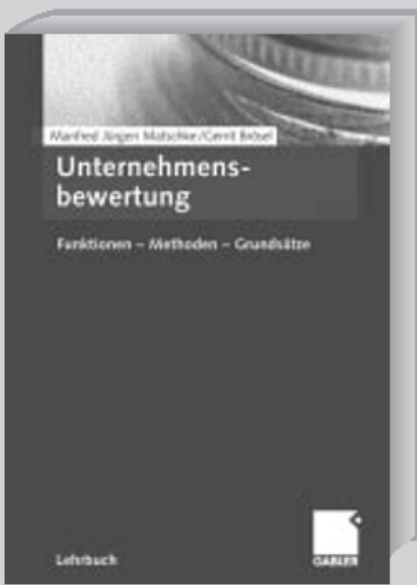

Manfred Jürgen Matschke/ Gerrit Brösel

\section{Unternehmensbewertung}

Funktionen - Methoden - Grundsätze 2005. XXXII, 713 S. Geb. EUR 44,90 ISBN 3-8349-0012-5

Inhalt: Grundlagen - Entscheidungsfunktion und Entscheidungswert Vermittlungsfunktion und Arbitriumwert - Argumentationsfunktion und Argumentationswert - Grundsätze der Unternehmensbewertung

Die Autoren: Prof. Dr. Manfred Jürgen Matschke ist Inhaber des Lehrstuhls für Allgemeine Betriebstrirtschaftslehre und Betriebliche Finanzwirtschaft, insbesondere Unternehmensbewertung an der Ernst-Moritz-Arndt-Universität Greifswald.

Dr. Gerrit Brösel ist Habilitand und Wissenschaftlicher Assistent am Fachgebiet Rechnungshesen/Controlling der Technischen Universität IImenau.

Siehe auch: whrns.konvergenz-management.com.

Änderungen worbehalten. Erhältlich im Buchhandel oder beim Verlag.

Abraham-Lincoln-Str. 46

65189 Wiesbaden

Tel: $06117878-626$

Fax: $06117878-420$

wwwgabler.de

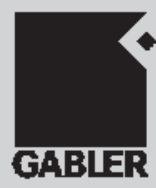

